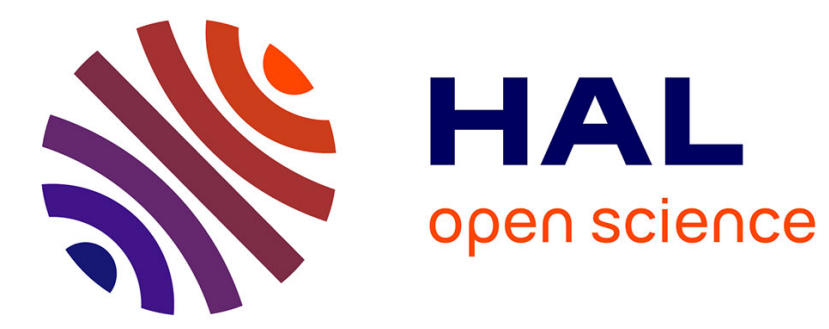

\title{
Une date consulaire (213 après J.-C.) sur un tuyau de plomb viennois \\ Jean Burdy, André Cochet
}

\section{To cite this version:}

Jean Burdy, André Cochet. Une date consulaire (213 après J.-C.) sur un tuyau de plomb viennois. Gallia - Fouilles et monuments archéologiques en France métropolitaine, 1992, 49, pp.89-97. 10.3406/galia.1992.2931 . hal-01915222

\section{HAL Id: hal-01915222 \\ https://hal.science/hal-01915222}

Submitted on 19 Jan 2020

HAL is a multi-disciplinary open access archive for the deposit and dissemination of scientific research documents, whether they are published or not. The documents may come from teaching and research institutions in France or abroad, or from public or private research centers.
L'archive ouverte pluridisciplinaire $\mathbf{H A L}$, est destinée au dépôt et à la diffusion de documents scientifiques de niveau recherche, publiés ou non, émanant des établissements d'enseignement et de recherche français ou étrangers, des laboratoires publics ou privés.

\section{(1) (1) $\$$}

Distributed under a Creative Commons Attribution - NonCommercial - NoDerivatives $\mid 4.0$ 


\title{
Une date consulaire (213 après J.-C.) sur un tuyau de plomb viennois
}

\author{
par Jean BURDY* et André COCHET**
}

Un fragment de tuyau de plomb romain de grand calibre, provenant à peu près certainement de la rive draìte du Rhône en face de Vienne (Isère), porte, outre une marque nouvelle d'un plombier viennois, une date consulaire. Il témoigne de grands travaux hydrauliques à des fins publiques sous Caracalla, en 213 après J.-C. Ce qui remet en question l'idée trop longtemps admise que le début du III ${ }^{\mathrm{e}}$ s. fut une période de déclin pour Vienne et vit le commencement de l'abandon du quartier de la rive droite.

A piece of a Roman lead pipe of a large bore, coming nearly certainly from the river Rhône right bank, opposite Vienne (Isère), bears, besides a Vienne plumber's new stamp, a consular date. It bears evidence of great hydraulic works for public purposes under Caracalla in A.D. 213. That brings in question the idea too long granted that the early third century was a period of decline for Vienne and saw the beginning of the desertion of the right bank district.

Mots clés : tuyau de plomb romain, marque de plombier, date consulaire, Caracalla, Vienne, Isère.

* 16, rue Roger-Radisson, 69005 Lyon.

** ERA 3, 7, rue Raulin, 69365 Lyon Cedex 07. 
Nos recherches sur les aqueducs romains nous ont fait retrouver fortuitement un fragment de tuyau de plomb portant deux estampilles, d'une part la marque d'un plombier viennois, d'autre part une date consulaire (fig. 1). Cette dernière confère à l'objet. un intérèt archéologique et historique de toute première importance.

\section{LE TUYAU}

Le fragment, de masse $48 \mathrm{~kg}$, provient d'un tuyau de grand module. Détaché par sciage, il se présente comme une plaque incurvée, longue de $90 \mathrm{~cm}$, large de 31 à $34 \mathrm{~cm}$, épaisse de 1,5 à $1,6 \mathrm{~cm}$, avec sur un bord longitudinal le cordon de soudure qui fermait le tuyau.

La restitution de la section complète du tuyau est possible à partir de la courbure du fragment, mais délicate du fait de sa légère ovalité (fig. 2). On peut estimer le diamètre intérieur moyen à $20 \mathrm{~cm}$ et le périmètre à environ $62 \mathrm{~cm}$.

Ces dimensions conduisent, avec le cordon de soudure, à une masse linéique de $120 \mathrm{~kg}$ par mètre, et à une masse de 360 à $400 \mathrm{~kg}$ pour un tuyau qui aurait la longueur classique de dix pieds. Notre tuyau se classe ainsi parmi les plus gros et correspond au calibre nonagenaria (tuyau de 90) de Frontin (cf. Annexe ${ }^{1}$. Lne aussi forte section parait incompatible avec une canalisation privée : il y a raison de pensere que le tuyau appartenait à une conduite publique.

Le cordon de soudure est de section rectangulaire ${ }^{2}$, large de $6,2 \mathrm{~cm}$ et épais de $1,4 \mathrm{~cm}$. Du côté intérieur, on observe que la soudure a rempli un

I Frontrix, Les aqueducs de la ville de Rome, texte établi, traduit ef commente par P. (irimal, Paris, Les Belles Lettres, 1961. IX, p. 28:- F. Kват7scinmer, La technique romaine. Bruxelles, 1966, р. 55: - - 11. Fанывusch, Über Abflussmessung und standardisierung bei den Wasserversorgungsanlagen Roms, in : Wasserversorgung im antiken Rom, Munich, 1982, p. 139-144; - P. PAcF, Cili acquedotti di Roma. Rome, 1986, p. 86-90; - A. Cochet, J. Hansen, Conduites et objets de plomb gallo-romains de lienne (Isere), 46 suppl. à Gallia. Paris ed. du (CNRS, 1986, p. 60, 61, 1.42. Notre tuyau a les dimensions du n" 49c du catalogue etabli par .J. Hansen : il sagit d'un exemplaire unique bien different des autres par son gros calibre.

? Le cordon de soudure est du type I I) défini par A. Cochet dans A. Cocmer, J. IIansen, op. cit. p. 29-32. Pour ce type de soudure. le tuyau, dispose horizontalement, etait rempli de sable, et sans doute enterré, deux règles déterminant une sorte de rigole de la largeur du cordon de soudure désiré. C'est dans cet espace qu était versé le métal dapport liquide (soudure "à la poche" dans le jargon d'atelier des fondeurs contemporains). intervalle d'environ $0,3 \mathrm{~cm}$ entre les bords non jointifs et légèrement dénivelés, d'environ $0,5 \mathrm{~cm}$, de la plaque cintrée (fig. 3). En passant à travers cet espace libre, le métal d'apport de la soudure longitudinale a formé localement à l'intérieur du tuyau un bourrelet, atteignant $0,5 \mathrm{~cm}$ d'épaisseur, épousant exactement les grains du sable de remplissage en certains endroits et formant, quelques centimètres plus loin une surface convexe, lisse (fig. 4a). Ces différences tiennent sans doute à la température de coulée de ce métal d'apport, assez chaud pour être parfaitement fluide, ou bien tout juste au-dessus de la température de solidification, et solidifié avant de parvenir tout à fait aux limites de l'espace offert. Là où le métal a bien pris l'empreinte de ce remplissage, il porte des lignes longitudinales, en creux, qui semblent correspondre à la trace laissée par un objet (peut-être un simple chiffon) introduit dans le jour subsistant entre les bords à assembler, et glissé tout au long du tuyau, sans doute afin d'éviter que du sable ne reste dans l'espace destiné à recevoir le métal d'apport (fig. 4b).

En dautres points, on observe de minces bavures s'étalant le long de la paroi jusqu'à plus de $5 \mathrm{~cm}$ de l'assemblage.

Ces indices montrent que le remplissage du tuyau au moment de la soudure n'était pas parfait.

Au demeurant ce fragment de tuyau est, par son découpage, une excellente pièce de démonstration pour l'explication des procédés de soudage des tuyaux romains.

L'objet est en bon état, avec seulement quelques traces de chocs ou de coups d'outils reçus soit dans sa période d'utilisation, soit au moment de son dégagement ou ultérieurement. Extérieurement, le métal est seulement terni, il n'est pas corrodé et ne présente pas non plus la couche superficielle blanchàtre des plombs ayant vieilli en ambiance calcaire. A l'intérieur l'eau na a pas laissé de dépôt.

\section{LES ESTAMPILLES}

Le fragment a été découpé pour la conservation des inscriptions, particulièrement de la plus grande, qui a retenu l'attention et qu'on peut effectivement qualifier de rarissime, l'autre étant d'un type commun. Toutes deux sont en relief et venues de fonderie à la fabrication.

La première est inscrite dans un cartouche rectangulaire long de $77 \mathrm{~cm}$ et large de $4,8 \mathrm{~cm}$ (dimensions extérieures) (fig. 5). Ce cartouche est légèrement en oblique par rapport au cordon de soudure, 


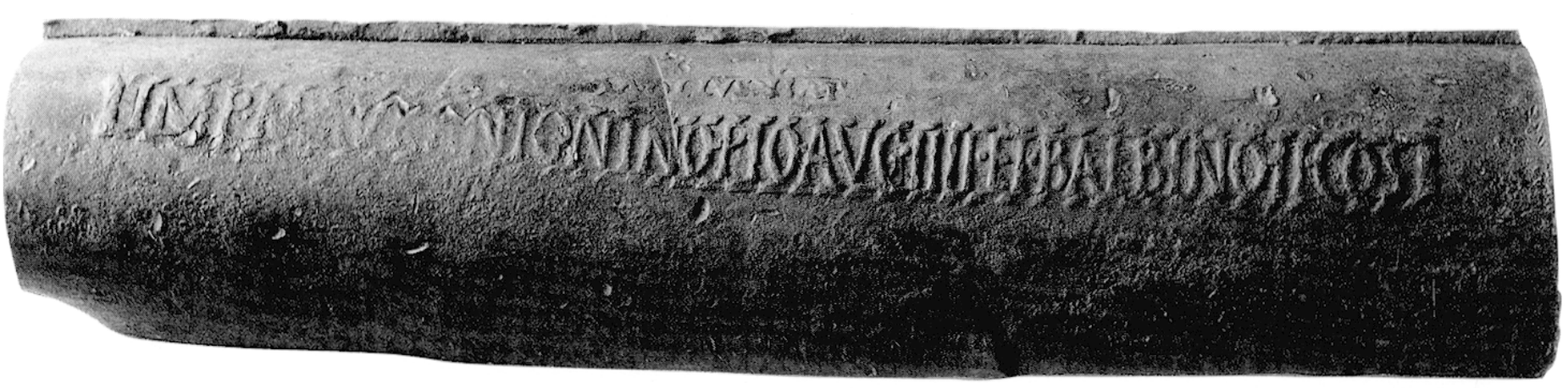

Fig. 1 - Vue d'ensemble de la face externe.

Fig. 2 - Perspective cavalière schématique du fragment conservé : en trait fin la partie manquante.

son angle supérieur gauche étant à $7,5 \mathrm{~cm}$ du bord de celui-ci, et son angle droit à $9 \mathrm{~cm}$. La seconde est dans un cartouche rectangulaire lui aussi, long de $13,5 \mathrm{~cm}$ et large de $2,4 \mathrm{~cm}$, parallèle au premier, audessus de lui, à $1 \mathrm{~cm}$ de bord à bord; elle est peu excentrée, de $3 \mathrm{~cm}$, et renversée.

\section{LA GRANDE ESTAMPILLE}

Le cadre, épais de $0,7 \mathrm{~cm}$, est d'aspect cordé sur les côtés longs, et devient continu aux extrémités. Les lettres sont hautes de 3,0 à $3,4 \mathrm{~cm}$, sans ligatures, relativement étroites du fait de la longueur du

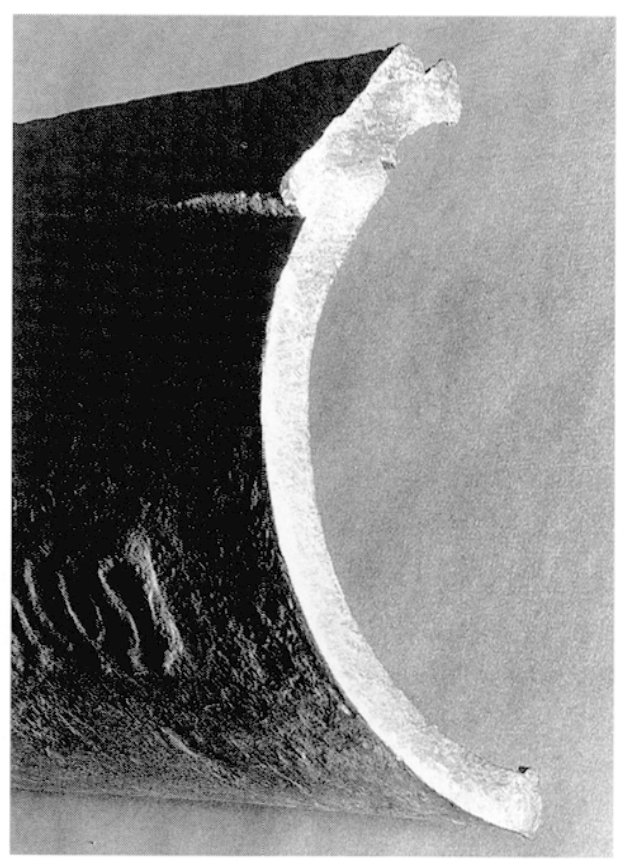

Fig. 3 - Extrémité droite du fragment : on remarque la dénivellation entre les deux bords de la feuille rapprochés pour former le tuyau. 


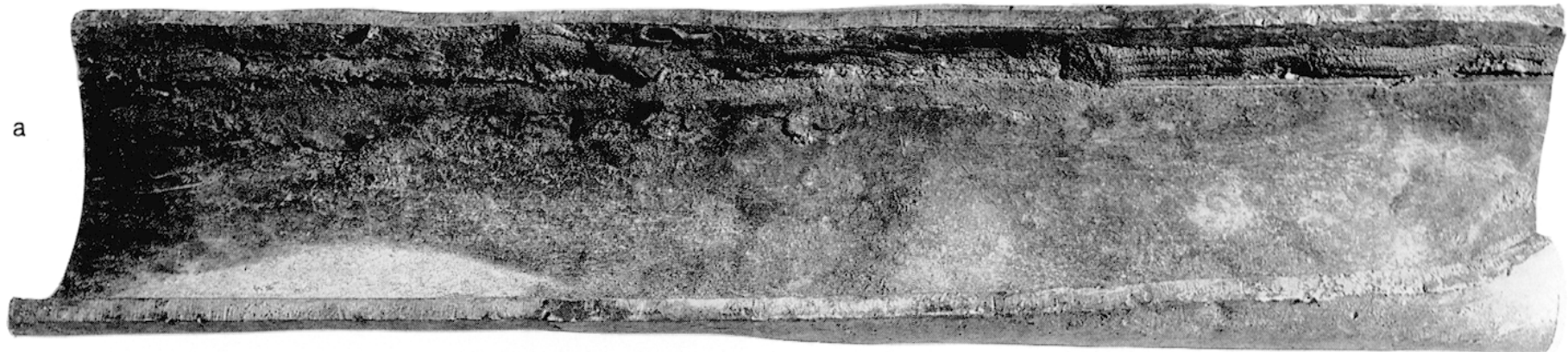

b

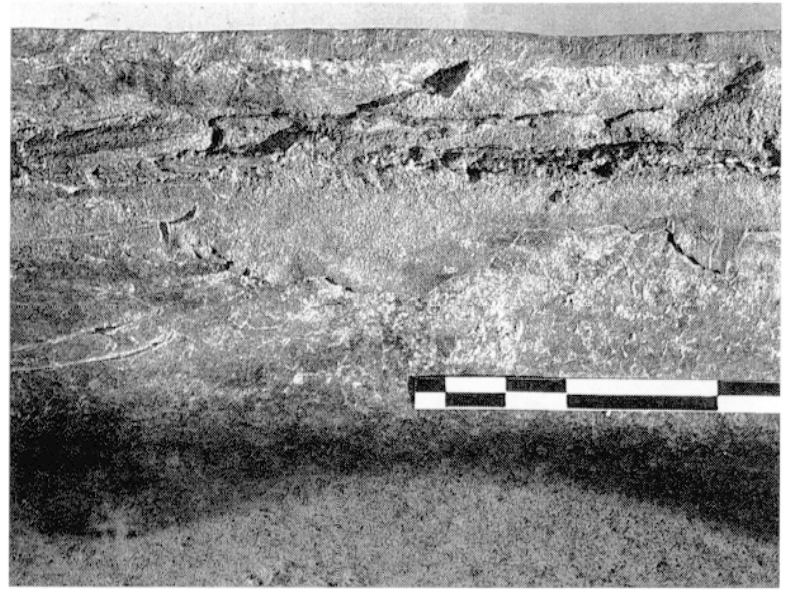

Fig. 4 - a, vue d'ensemble de la face interne;

b, face interne: région de la soudure avec des bavures formées par le métal d'apport entre la face interne et le remplissage de sable mis en place pour la soudure.

texte. Celui-ci, aux mots séparés par des points à mi-hauteur, ne présente aucune difficulté de lecture, bien que partiellement mal venu de fonderie, avec un très faible relief, sur $10 \mathrm{~cm}$ de long.

\section{IMP.M.AVR·ANTONINO·PIO·AUG IIII.ET.BALBINO.II.COS}

Il s'agit d'une date consulaire et c'est l'ablatif qu'il faut retenir:

Imp(eratore) M(arco) Aur(elio) Antonino Pio Aug(usto) IIII et Balbino II co(n)s(ulibus).

Sous le quatrième consulat de l'empereur Marcus Aurelius Antoninus Pius Auguste et le deuxième consulat de Balbinus.

Le tuyau est donc parfaitement daté, de la troisième année du règne de Caracalla, en 213 après J.-C. Cette épigraphe est la seconde du genre, sur un luyau de plomb, trouvée hors de Rome. La première, trouvée en 1899 à Chester, en Grande-Bretagne, mentionne le neuvième consulat de Vespasien et le septième de Titus. Elle provient du système d'alimentation en eau du fort de Deva ${ }^{3}$.

A Rome, Lanciani a relevé dix inscriptions donnant une date consulaire sur des tuyaux de plomb. Dressel en a rejeté une, de lecture à son avis erronée, il en a ajouté deux, doublant des inscriptions déjà données par Lanciani, et trois nouvelles ${ }^{4}$. Au total, six sont d'un libellé comparable à celui qui nous occupe, c'est-à-dire limité à la date; les cinq autres sont beaucoup plus longues et détaillées. Une inscription se rapporte à Hadrien (121 après .J.-C.), deux à Marc Aurèle (161 et 175 après J.-C.) et trois à Commode $(177,179$ et 183 après J.-C.), les autres à Septime Sévère $(201,202$ et 203), Élagabal (219) et Sévère Alexandre (233). A part la première, elles concernent donc la fin $d u I^{\mathrm{e}}$ s. et le début du $\mathrm{II}^{\mathrm{e}}$, sous les derniers Antonins et les Sévères, c'est-à-dire l'une des deux périodes les plus riches de l'épigraphie datée de l'alimentation en eau de Rome ${ }^{5}$. D'après Lanciani, toutes, à l'exception d'une seule datant de Commode (179), marquent des canalisations publiques pour des camps militaires et, plus précisé-

3 II. TAYLOR, Four recent discoveries of Roman remains in the city of Chester, in : Proceedings of the Sociely of Antiquaries of London, 2 S. 18, 1900, p. 91-98; - H. Dessau. Inscriptiones latinae selectae, II-II, 8704a; - I. KFPPIE, Linderslanding Roman Inscriplions, Londres, 1991, p. 27.

Le musée de Vichy conserve aussi un tuyau de plomb daté de la huitième année sous les empereurs Septime Sévère et Caracalla. Mais, outre qu'il est d'origine douteuse, sa marque ne se présente pas sous la forme d'une véritable date consulaire et nous le laissons de còté. Cf. Année épigraphique, 1945, p. 43 (138); - A. Mord.ft, Vichy gallo-romain, Mâcon, 1957, p. 2332:34: - J. Cornochrn, Vichy antique, Clermont-Ferrand, 1981. p. 111.

4 R. Lanciani, Topografia di Roma antica. I comentarii (sic) di Frontino intorno le acque e gli acquedotti. Silloge epigrafica aquaria, Rome, 1881, p. 429, 439, 440, 461 ( $\mathrm{n}^{\text {os } 34,106,}$ $108-112,114,116,255)$; - H. Dresset, Corpus Inscriptionum Latinarum, XV, p. 911 sq. (7240-7242, 7319, 7359-7366). « Non multae fistulae consulum nota signatae sunt.".

5 R. Lanciani, op. cil., p. 412. 


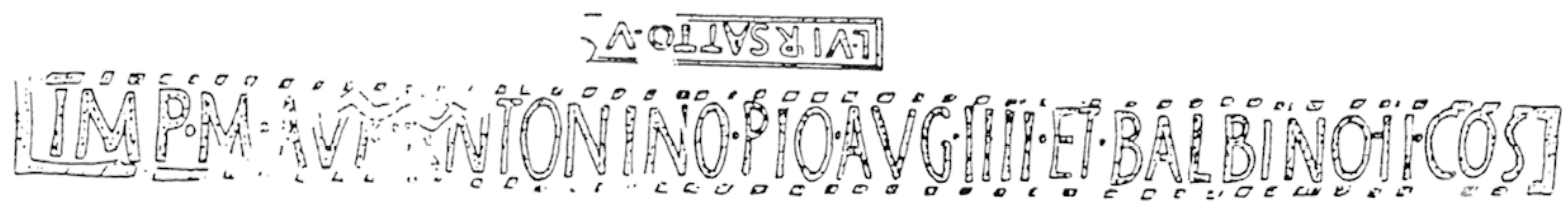

0 $20 \mathrm{~cm}$

Fig. 5 - Les inscriptions d'après un frottis : la ligne SS correspond au bord du cordon de soudure, elle précise le placement des cartouches (position normale pour la marque impériale et renversée pour la marque de fabrique).

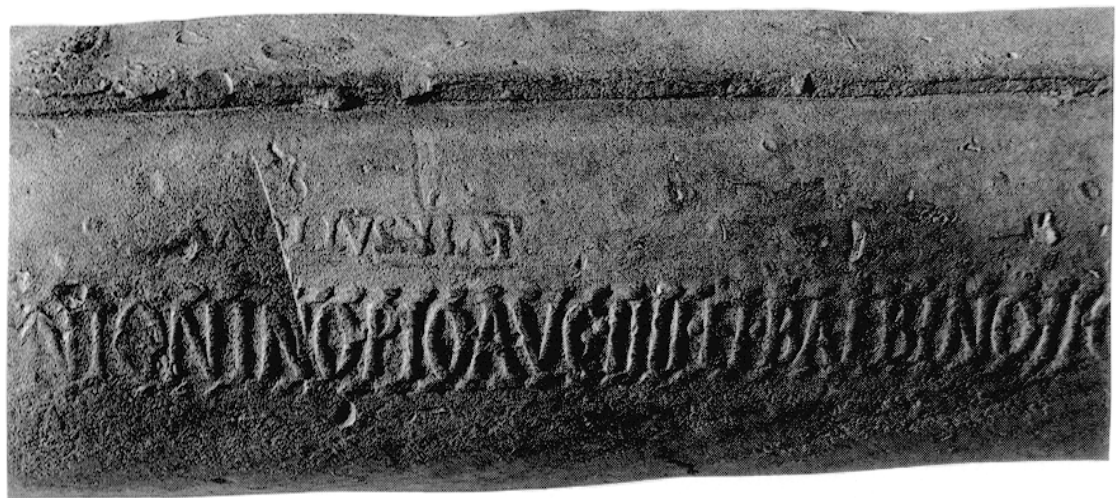

liig. 6 -

Détail de la partie centrale

avec l'inscription

L.VIR SATTOVV

placée à l'envers par rapport

à la marque impériale ment même, le camp prétorien ${ }^{6}$. Le même auteur signale en outre un "tubo grandissimo", centenaria, marqué du nom d'un curateur des eaux ayant effectué des travaux hydrauliques pour le compte de Caracalla ${ }^{\text {? }}$.

Dressel ne retient que cinq attributions assurées pour l'administration publique. Néanmoins, le grand calibre de notre tuyau faisant déjà penser à une conduite publique, nous trouvons cette hypothèse singulièrement renforcée, et par la date consulaire, et par les données provenant de Rome ${ }^{8}$.

6 On trouve un résumé de la question dans E. DE Ri (a(iIfro, Dizionario Epigrafico di Antichita Romane, Rome. 1895. au $\S$ "Date consolari", p. 586 .

7 R. Lancian, op. cil.. p. 440.

8 Cette hypothèse est aussi en accord avec les constatations de $Y$. Burnand. dans La documentation épigraphique sur les aqueducs de la Gaule et de la Germanie romaines, Journées d'études sur les aqueducs romains, Lyon (26-28 mai 1977), Paris, 1983. p. 51-73. Sur les quatorze textes retenus, tous lapidaires, cing sont exactement datés par l'indication de l'année consulaire. La grande majorité se rapporte à des travaux d'adduction dans l'intèrèt d'une collectivité, soit militaire, pour un castellum du limes. soit civile, pour un vicus ou le plus souvent un chef-lieu de cité.

\section{LA PETITE ESTAMPILLE}

Le cadre est un filet simple, continu, épais de $0,2 \mathrm{~cm}$. Les lettres sont hautes de $1,3 \mathrm{~cm}$ à $1,5 \mathrm{~cm}$, et proportionnellement bien plus larges que dans la grande inscription (fig. 6).

On lit :

\section{L.VIR.SATTO $V$}

qui est la marque du plombier, au nominatif :

L(ucius) Vir(eius ou ius) Satto V(iennae fecit).

L'inscription devient évanescente vers la fin, où le cadre disparaît. Il est certain qu'il n'y a pas de ligature $\mathrm{F}$, mais un $\mathrm{F}$ séparé du $\mathrm{V}$ ne serait pas impossible, l'empreinte ètant incomplète. Précisons que, à ce jour, on ne connaît aucune marque viennoise avec la lettre $\mathrm{V}$ seule.

Ceci mis à part, cette estampille, d'un type ordinaire, a pour intérêt de nous faire connaître une marque d'artisan nouvelle, avec l'avantage rarissime d'être parfaitement datée. Le gentilice peut être le même que celui de la marque connue T. Vir. Fortunatus. V.F., c'est-à-dire Vireius, ou bien Virius. Le cognomen Satto s'est déjà rencontré en quatre exemplaires à Vienne, mais toujours associé à Senter dans 
la marque SATTO ET SENTER V F; un exemplaire a été trouvé à Saint-Romain-en-Gal, un autre à Sainte-Colombe ${ }^{9}$.

\section{LE CONTEXTE ARCHÉOLOGIQUE}

L'objet est, en lui-même, d'un intérêt insigne, et il faut se féliciter qu'il nous soit parvenu, mais nous devons en même temps déplorer - c'est un euphémisme ... - les circonstances entourées d'inconnues de sa remise au jour.

\section{ORIGINE DE L'OBJET}

C'est en janvier 1990 que nous avons appris son existence dans une propriété de Saint-Didier-auMont-d'Or ${ }^{10}$. Il provient d'un lot de dix tonnes de plomb de récupération livré en 1980 à une entreprise de recyclage de vieux métaux de la banlieue lyonnaise. L'identité des fournisseurs, occasionnels, n'a pas été relevée, et tous nos efforts pour remonter à la source de la découverte sont malheureusement restés vains. Il nous a été dit que ces tuyaux de plomb auraient été trouvés au bord du Rhône, rive droite, en face de Vienne. Indication probablement véridique, ne laissant guère place au doute, mais regrettablement imprécise ${ }^{11}$. A quelle hauteur de SaintRomain-en-Gal ou de Sainte-Colombe a-t-elle été trouvée? Quelle était la direction de la canalisation in silu? Etait-elle, plus ou moins, perpendiculaire ou parallèle au Rhône, el à quelle distance de lui? Quelle a été la longueur récupérée? Nous n'avons pu savoir dans quelle proportion les tuyaux romains intervenaient dans lès dix tonnes livrées à l'usine. La totalité de cette masse correspondrait à quelque vingt-sept tuyaux de dix pieds, soit une longueur d'environ quatre-vingts mètres, chiffres qui, bien que devant certainement être revus à la baisse, peuvent néanmoins être de l'ordre de grandeur de la quantité récupérée. L'information qui nous a été donnée, selon laquelle les tuyaux ont été coupés en longueurs de $1 \mathrm{~m}$ pour être mis au four de fusion, conduit à penser que la longueur totale était importante, et il est indubitable que le fragment conservé n'en est qu'une toute petite fraction.

9 A. Cochet, J. HANSEN, op. cit., p. 128-130, 158, 159.

10 . Vous remercions $M$. Sciandra de nous avoir donné toutes facilités pour l'ètude du tuyau.

11 La fin des années 1970 fut une période de grands travaux publics sur la rive droite : autoroute, aménagement de la rive du fleuve par la Compagnie nationale du Rhône, lycée. etc.; les travaux privés ne doivent pas ètre laissés de còté.

\section{UNE ALIMENTATION PUBLIQUE DE LA RIVE DROITE}

La date consulaire et la grande section du tuyau indiquent, nous l'avons dit, qu'on a affaire à une canalisation publique ${ }^{12}$. Et il est extrêmement probable que le plomb provienne effectivement de la rive droite du Rhône, en face de Vienne, ce que nous admettrons pour la suite. Or, bien que le grand chantier de fouille de Saint-Romain-en-Gal ait révélé un réseau de distribution d'eau considérable, deux fontaines publiques, un petit établissement de bains, de riches demeures et une zone artisanale certainement copieusement desservies, bien qu'on connaisse en outre des thermes importants à Saint-Romain (Palais du Miroir), et d'autres à Sainte-Colombe, jusqu'à maintenant on ignore tout de l'adduction d'eau pour cette région, aucune trace d'aqueduc n'ayant jamais été repérée.

Notre tuyau, témoin de travaux importants, apporte une lueur sur le problème. Apport toutefois peu circonstancié, au point qu'on est réduit à de fragiles conjectures, et surtout à poser des questions. La canalisation dont il faisait partie était-elle particulièrement destinée à l'alimentation d'un établissement gros consommateur d'eau, comme des thermes, ou bien à tout un quartier, voire à l'ensemble de la rive droite? Était-ce la canalisation principale d'amenée, ou seulement une extension du réseau de distribution? D'où venait-elle? Etait-elle alimentée par des sources de la rive droite, ou plutôt amenaitelle de l'eau fournie par des aqueducs de la rive gauche? Traversait-elle le Rhône sur un pont, ou en conduite forcée reposant au fond du fleuve, à l'instar du siphon partant d'Arles pour alimenter le faubourg de Trinquetaille sur l'autre rive du Rhône ${ }^{13}$ ?

\section{L'APPORT HISTORIQUE}

Tirer toutes les conséquences historiques de l'existence de ce tuyau daté de l'an 213 n'est pas de notre compétence et, laissant cette tâche aux historiens, nous ne ferons qu'évoquer rapidement la question.

Caracalla, fils de Septime Sévère, est né à Lyon en 188 . Son règne, bien que bref (211 à 217), est mar-

12. J. Ilansen s'était étonné "que l'on ne trouve pas plus de traces dinscriptions rapportant que les tuyaux sont fabriqués pour le compte des autorités publiques", dans A. Соснгт et J. IIAssex, op. cil., p. 79.

13 L. A. Constavs, Arles antique, Paris, 1921, p. 400; - A. Grenifr, Manuel d'archéologie gallo-romaine, IV, Les monuments des eaux, I, Paris, 1960, p. 84-85 
qué par des innovations importantes dans tous les domaines, légal et administratif, financier, militaire, urbanistique. Rome, qui voit se réaliser de grands travaux sous les Sévères, doit en particulier à Caracalla des thermes grandioses, les plus grands pour l'époque, et un aqueduc, l'Aqua Antoniniana, pour les alimenter à partir de l'Aqua Marcia. Caracalla a traversé la Narbonnaise, en 213 précisément, pour gagner la Germanie : il a certainement dû passer par Vienne ${ }^{14}$

Pendant cette période, l'histoire de Vienne est des plus mal connues. Mis à part le socle d'une statue portant une longue dédicace à Caracalla, trouvé en 1853 dans les terrassements du chemin de fer, quartier Saint-Gervais ${ }^{\mathbf{1 5}}$, "le $1 \mathrm{I}^{\mathrm{e}}$ siècle est, en effet, caractérisé par une pénurie de documents tant épigraphiques qu'archéologiques, cette pénurie étant indiscutablement le signe d'un repli sur soi [...]. La citè avait abandonné dès la fin du II $^{\mathrm{e}}$ siècle le quartier méridional de la rive gauche, au début du $111^{\mathrm{e}}$ siècle le quartier de la rive droite" ${ }^{16}$. A SaintRomain-en-Gal, à en croire les résultats des fouilles menées jusqu'à maintenant, "le début du $\|^{e}{ }^{e}$ siècle marque un arrêt dans le développement du quartier et le début de son abandon. Dès lors, il semble que l'on ne construise plus et que l'on se contente de réoccuper partiellement les édifices du ${ }^{1}{ }^{\mathrm{e}}$ siècle " ${ }^{17}$.

Ces conceptions doivent être revues, pour le moins en ce qui concerne les premières décennies du III ${ }^{\mathrm{e}}{ }^{18}$. Notre tuyau témoigne que des travaux publics considérables ont été exécutés pour l'adduction d'eau en 213, sous Caracalla, sur la rive droite. Le déclin du site a peut-être commencé peu après, mais cette date de 213 doit être considérée comme appartenant encore à une période de prospérité et de développement.

14 P. Prtit, Histoire générale de l'Empire romain, Paris, 1974, p. 330, 331 ; - J. LF Gall, M. Le Glay, L'empire romain, I, Paris, 1987, p. 578-591; - L. Номо, Rome impériale el l'urbanisme dans l'antiquité, Paris, 1971, p. 314; II. Halmann, Itinera principum, Geschichte und Typologie der Kaiserreisen im Römischen Reich, Stuttgart, 1986, p. 223-226. Nous remercions $F$. Richard pour cette référence.

15 A. Al.t.mer, A. de Terrebasse, Inscriptions de Vienne, 1875-1876, Inscriptions antiques et du Moyen-Age de Vienne en Dauphiné, I, p. 104-108; - CIL XII, 1851. 487.

16 A. Pelletier, Vienne antique, Roanne, 1982, p. 101,

17 C. Laroche, H. Savay-Gugrraz, Un quartier de Vienne antique sur la rive droite du Rhône, in : SaintRomain-en-Gal, Paris, 1984, Guides archéologiques de la France, $n^{\circ} 2$, p. 36 .

18 C'est bien ce qu'Anne LF Bot-Heil.y et B. Helt.Y ont fait dans : La fouille de la place Camille-Jouffray, in :

\section{CONCLUSION}

La découverte de ce tuyau de plomb viennois, de grand calibre et daté, offre un bilan contrasté.

D'un côté, elle fournit un document d'importance touchant à une question encore très obscure, à savoir l'adduction publique d'eau sur la rive droite. Ce document parfaitement daté, dans une période historique mal connue, doit conduire à un réexamen de celle-ci.

Par ailleurs, une bonne part des informations susceptibles d'être apportées par l'objet ont été perdues du fait d'une récupération clandestine, à l'insu des compétences archéologiques. Cette circonstance est éminemment déplorable, mais n'a malheureusement rien d'exceptionnel. Les métaux sont des matières facilement recyclables, et de tous temps les objets métalliques ont été récupérés et fondus pour en fabriquer de nouveaux. C'est ainsi que les objets du passé ont, en quasi-totalité, disparu. Et il en est aujourd'hui de même qu'hier : la moitié de la production française actuelle de plomb provient de la récupération de débris, de déchets et de résidus d'origines diverses ${ }^{19}$. Dans la quantité, il se trouve à coup sûr des éléments qui auraient mérité un sort meilleur qu'une disparition dans un four. C'est pourtant, nous a-t-on dit, ce qui est arrivé, en 1950, pour la totalité de deux tonnes de tuyaux de plomb romains provenant de la région lyonnaise ${ }^{20}$. Ainsi, on peut apprécier à sa juste valeur la conservation de ce fragment de tuyau viennois daté.

Il convient toutefois de redoubler d'attention, et d'agir pour l'information de tous et la prise de conscience la plus large, afin d'éviter le plus possible, à l'avenir, des destructions telles que celles ici évoquées. On peut raisonnablement espérer que de nouvelles découvertes, pleinement exploitées, viendront répondre à nombre des questions que nous nous posons.

Jean Burdy et André Cochet

F. Baratte et alii, Le trésor de la place Camille-Jouffray à Vienne (Isère), Un dépôt et son contexte archéologique, $50^{\mathrm{e}}$ suppl. à Gallia, Paris, Éd. du CNRS, 1990, p. 9-29. Si tous les quartiers extra muros de Vienne ont èté abandonnés à la fin de l'Antiquité, ils ne l'ont pas été simultanément. "La désertification est progressive. Datée en général du milieu du $\mathrm{HI}^{\mathrm{e}}$ siècle sur la rive gauche (...), elle ne paraît pas antérieure à la fin du III $^{\mathrm{e}} \mathrm{s}$. au plus tôt et au milieu du $\mathrm{Iv}^{\mathrm{e}} \mathrm{s}$. au plus tard, place Camille-Jouffray".

$19250000 \mathrm{t}$ par an, pour moitié seulement en métal de première fusion élaboré à partir du minerai. Le plomb vaut actuellement, en $1990,4000 \mathrm{~F}$ la tonne.

20 Le fait est avéré. Nous tenons le renseignement de M. Bonniel (Saint-Didier-au-Mont-d'Or). 


\section{ANNEXE}

\section{OCTOGENARIA OU NONAGENARIA?}

Les figures 7 et 8 représentent la section du fragment de tuyau conservé, section relevée à l'extrémité droite, la mieux conservée.

Sur ces dessins figure un réseau de cercles correspondant aux mandrins sur lesquels étaient formés les tuyaux de $60,70,80$ et 90 définis par Frontin (nos LIV, LVI, LVIII. LX). Nous avons omis les dimensions intermédiaires que Frontin qualifie de "non usitées".

Les différents traducteurs et commentateurs s'accordent sur l'interprétation des dimensions (valeurs données par Kretzschmer ${ }^{21}$, mais aussi par Gri$\left.\mathrm{mal}^{22}, \ldots\right)$.

On voit combien il est difficile de faire coïncider la forme réelle d'un objet avec des formes géométriques théoriques.

La partie MP de la section, mis à part un petit accident en $\mathrm{M}$ dù à une déformation récente, s'accorde à peu près aussi bien avec le rayon du tuyau de 80 qu'avec celui de 90 , mais elle est manifestement trop ouverte pour correspondre aux dimensions inférieures.

Au voisinage de $P$, un pliage assez marqué relie cet arc régulier à une zone $\mathrm{PN}$ pour laquelle la courbure est bien plus faible. Dans la portion NQ nous trouvons une partie accidentée, correspondant au métal d'apport de la soudure. Les bords à relier n'étaient pas jointifs et ne se trouvaient pas au même niveau.

L'existence d'un petit pan, presque plat, correspondant à la partie PN s'explique par le fait que lors d'un cintrage sur un mandrin, il est impossible, par liig. 7 -

Coincidence de la section relevée avec le profil theorique du nonagenaria de Frontin.

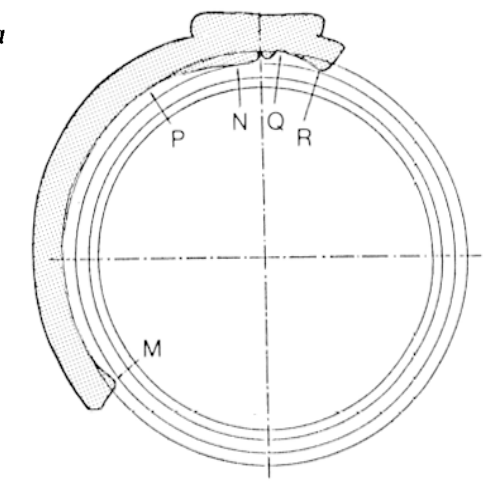
$10 \mathrm{~cm}$

Fig. 8 -

Coincidence de la section relevée avec le profil theorique de l'octogenaria de Frontin.

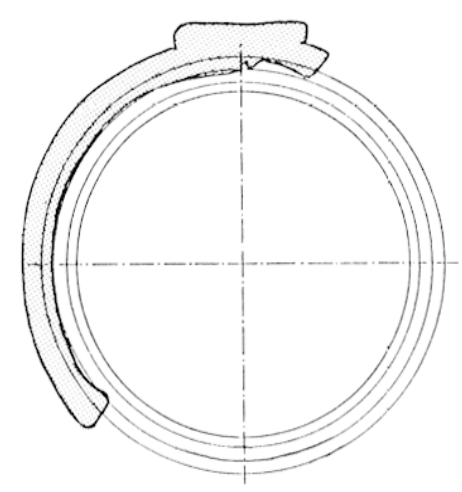
$10 \mathrm{~cm}$ 


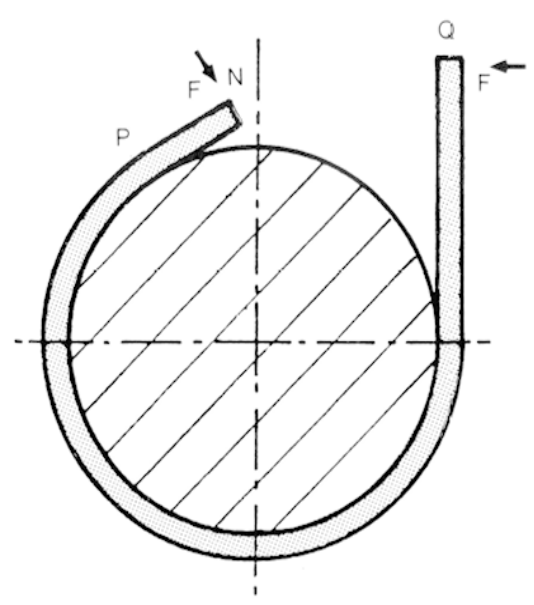

a

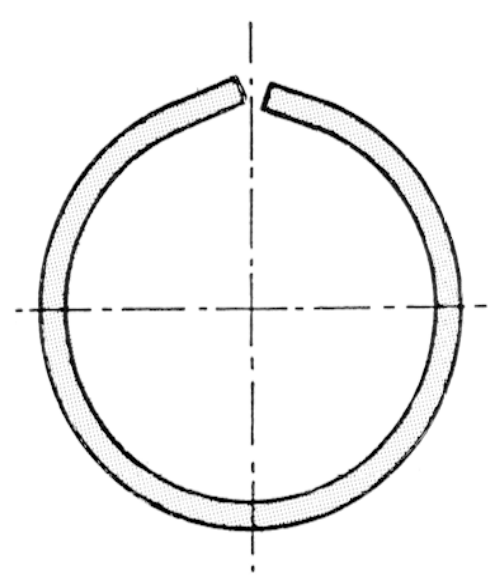

b

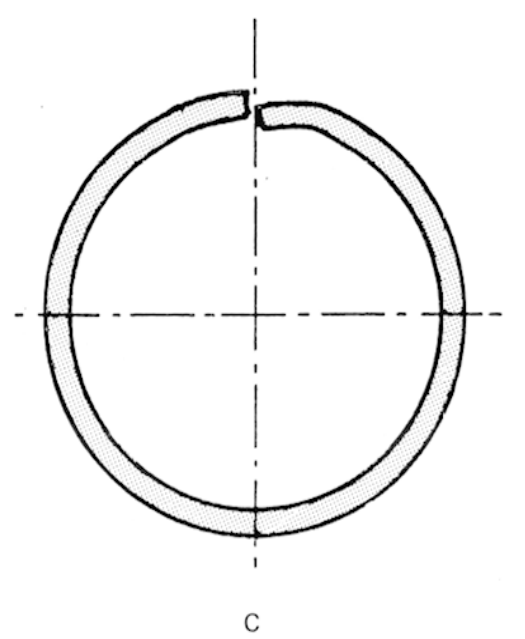

simple pression, d'appliquer parfaitement le bord d'une feuille sur le mandrin, la figure $9 \mathrm{a}$ en montre la raison : au fur et à mesure que l'on roule la feuille sur le mandrin, la force $\mathrm{F}$ appliquée au bord de la plaque à cintrer s'exerce sur un bras de levier PN qui va en décroissant. Pour obtenir un plaquage parfait il faudrait un martelage de la face extérieure du tuyau, allongeant le métal, véritable travail de chaudronnerie. Une telle opération aurait écrasé les inscriptions moulées lors de la coulée de la feuille de métal; ces inscriptions étant intactes nous sommes sûrs qu'il n'y a pas eu de martelage. On a donc retiré le mandrin alors que le cylindre n'était pas complètement formé (fig. 9b) puis enfoncé les deux pans peu ou pas cintrés pour obtenir le cylindre très approximatif qu'on observe (fig. 9c).

Toutes ces approximations à la fabrication rendent incertaine l'attribution de notre fragment à un module précis de Frontin. En parlant de "nonagenaria" nous adoptons la solution la plus probable, mais, vu les assez larges tolérances de fabrication observées sur d'autres canalisations, un "octogenaria" n'est pas impossible, ni mème un des calibres qualifiés d'inusités par Frontin comme le tuyau de 85 .

J. B. et A. C.

Fig. 9 -

a, cintrage d'une feuille de plomb sur un mandrin;

$\mathrm{N}$ et $\mathrm{Q}$, bords de la feuille;

$\mathrm{F}$, force appliquée à la feuille ;

FP. bras de levier dont dépend l'efficacité du cintrage (en fin de travail FP qui ne peut dépasser la valeur P. tend vers zéro);

b, la feuille imparfaitement formée au retrait du mandrin ; c, les bords sont renfoncés vers l'intérieur du tuyau avant de passer à l'opération de soudure. 